\title{
Die Zivilgesellschaft als Hauptakteur der Informationsgesellschaft
}

Adama Samassekou

\section{(2) OpenEdition}

\section{Journals}

Electronic version

URL: http://journals.openedition.org/sjep/571

DOI: 10.4000/sjep.571

ISSN: 1663-9677

\section{Publisher}

Institut de hautes études internationales et du développement

\section{Printed version}

Date of publication: 1 novembre 2003

Number of pages: 229-231

ISSN: 1660-5926

\section{Electronic reference}

Adama Samassekou, "Die Zivilgesellschaft als Hauptakteur der Informationsgesellschaft », Schweizerisches Jahrbuch für Entwicklungspolitik [Online], 22-2 | 2003, Online erschienen am: 10 Juni 2010, abgerufen am 08 September 2020. URL : http://journals.openedition.org/sjep/571 ; DOI : https://doi.org/10.4000/sjep.571 


\title{
Die Zivilgesellschaft als Hauptakteur der Informationsgesellschaft
}

\author{
Adama Samassekou*
}

ch wurde gebeten, auf den Artikel im vorliegenden Werk über die Beteiligung der Zivilgesellschaft am Weltgipfel über die Informationsgesellschaft (WSIS) zu reagieren. Für mich bedeutet dies eine besondere Freude, denn als Mitglied der Zivilgesellschaft fühle ich mich direkt angesprochen. Dass eine Person, die sich gegenwärtig in den Netzen der Zivilgesellschaft in Afrika und anderswo aktiv engagiert, in das Amt des Vorsitzenden des Vorbereitungsausschusses für den Weltgipfel über die Informationsgesellschaft (WSIS) berufen wird, ist an sich ein starkes Zeichen und bietet eine begrüssenswerte Gelegenheit, um zusammen mit allen betroffenen Akteuren einen einschliessenden, facettenreichen und offenen Prozess zu konzipieren. In der Folge möchte ich auf die Hauptmerkmale des Prozesses eingehen und meine persönliche Sichtweise dazu vorstellen.

\section{Der Gipfel einer neuen Generation}

Der Weltgipfel über die Informationsgesellschaft gehört zwar zur Serie der grossen Veranstaltungen der UNO, unterscheidet sich davon aber in vielfacher Hinsicht. Deshalb möchte ich die Idee des „Gipfels einer neuen Generation“ vorstellen.
Die ausschliesslich auf die Regierungsdelegierten beschränkten UNOGipfel stellten definitionsgemäss zwischenstaatliche Prozesse dar. Bislang begründeten diese Prozesse die Legitimation der Gipfeltreffen und gewährleisteten (im Regelfall) die Einhaltung der Verpflichtungen. Die Öffnung hin zur Zivilgesellschaft und zum Privatsektor bietet künftig solidere Garantien, weil alle direkt beteiligten Akteure einbezogen werden, und erlaubt es, die Rolle des Staates als bevorzugter Schlichter zwischen den Interessen der wirtschaftlichen Kräfte und den Erwartungen des Bürgers neu zu festigen.

Die Öffnung setzt Anpassungen voraus, die leichtfallen, da grosse Erwartungen gehegt werden, wenn neue Akteure zum Verhandlungstisch zugelassen werden.

Der erste Vorbereitungsausschuss (PrepCom1) befasste sich im Wesentlichen mit den Verfahren, welche die Schaffung eines Vertrauensverhältnisses ermöglichen sollten. Die Vertreter von 192 Staaten sollten sich auf die Modalitäten der Beteiligung der Beobachter verständigen, zu denen die Vertreter des Privatsektors, der zwischenstaatlichen Organisationen und insbesondere der Zivilgesell- 
schaft zählen - tausende von Organisationen, die alle ihre Anliegen vortragen möchten.

Zahlreiche Hürden behindern den Prozess. Es wäre sinnlos, die Augen davor zu verschliessen. Der Zeitfaktor bleibt die grösste Einschränkung. Alle Akteure haben knapp über ein Jahr Zeit, um sich $\mathrm{zu}$ informieren, $\mathrm{zu}$ mobilisieren, $\mathrm{zu}$ organisieren, Vorschläge $\mathrm{zu}$ komplexen Themen $\mathrm{zu}$ unterbreiten, sie zu vertreten und die Verhandlungen in Angriff zu nehmen.

\section{Büro der Zivilgesellschaft}

Bereits zu Beginn des Prozesses zeigte sich die Notwendigkeit, die Beteiligung der Zivilgesellschaft zu strukturieren, um den Dialog mit den Regierungen zu fördern. Dabei handelte es sich um eine unerlässliche Voraussetzung für die reelle und effektive Beteiligung der Zivilgesellschaft an der Ausarbeitung der Erklärung und des Aktionsplanes. Die Schaffung eines Mechanismus, der für ein gegenseitiges Vertrauensverhältnis unabdingbar ist, war mein aufrichtiger Wunsch. Auf Betreiben des Exekutivsekretariats des WSIS hat die Zivilgesellschaft mit dem Civil Society Bureau $C S B$ (Internationales Büro der Zivilgesellschaft) einen Mechanismus geschaffen, um ihre Vertretung in der Diskussion zu organisieren.

Es wurden verschiedene Massnahmen getroffen, um der Zivilgesellschaft und dem Privatsektor $\mathrm{zu}$ ermöglichen, Vorschläge $\mathrm{zu}$ unterbreiten, sie vor den Regierungen mündlich $\mathrm{zu}$ vertreten und an den Plenardiskussionen teilzunehmen mit dem Ziel, die verschiedenen Standpunkte besser zu verstehen.
Das Büro der Zivilgesellschaft verleiht der Zivilgesellschaft eine interne Legitimation und gewährleistet die Vertretung aller Familien der Zivilgesellschaft. Das erste Treffen zwischen den Vertretern der beiden Büros (Zivilgesellschaft und Regierungen) gilt als Premiere in der Geschichte der Vereinten Nationen und bildete ein herausragendes Ereignis, das sämtliche Teilnehmer als historisch bezeichneten.

Das Büro stellt meines Erachtens ein viel versprechendes Instrument dar: Es wird von Anfang an in den Dienst der Beteiligung der Basisgruppen gestellt und dient nicht nur als Struktur für verschiedene Individuen. Darin liegt der Sinn des Wortes „Familie“. Aus diesem Grund sind die sechzehn Familien des Büros wichtig. Sie stehen für die vielfältigen Visionen der Zivilgesellschaft zur Gesellschaft von morgen: die Sicht des Akademikers, des HighTech-Spezialisten, der NGO, der Jugendlichen, der Medien, der Gewerkschaften, der Frauen, der autochthonen Bevölkerungen, der Think-Tanks usw. Es ist entscheidend wichtig, dass das Büro der Zivilgesellschaft auch die regionale Repräsentativität berücksichtigt, um die je nach Kulturen unterschiedlichen Weltanschauungen einander gegenüberzustellen.

Das CSB muss als Sprachrohr für die Aspirationen der Zivilgesellschaft zu sämtlichen in der Millenniumserklärung aufgelisteten Themen von globalem Interesse noch gefestigt werden. Es erlaubt der Zivilgesellschaft, die Grundsätze und Massnahmen, die als geeignetste Reaktion auf die wichtigen aktuellen Anliegen betrachtet werden, auf abgestimmte Weise vor- 
zuschlagen, und spielt damit eine notwendige Rolle für die Einrichtung von globalen „Schaltstellen“ zwischen den Sphären der internationalen Diskussion und den Akteuren vor Ort. Deshalb bildet die Schaffung des CSB eine grundlegende Etappe beim Aufbau einer dynamischen Partnerschaft zwischen den Regierungen, der Zivilgesellschaft, dem Privatsektor und den zwischenstaatlichen Organisationen auf internationaler Ebene. Diese Partnerschaft - die Vorstufe zu einer neuen „Global Governance“ - stellt ein signifikantes Merkmal des Gipfels dar.

Soweit die Diskussion bei allen Akteuren ein deutlicheres Bewusstsein für die Anliegen gefördert hat, ist acht Monate vor dem Fälligkeitstermin - dem ersten Teil des Gipfels im Dezember 2003 in Genf - festzustellen, dass die Ergebnisse bereits sehr erfreulich aussehen.

\section{Offene Perspektiven}

Der eigentliche Gipfel in Genf und in Tunis bildet keinen Selbstzweck, sondern den Ansatz eines Prozesses, mit welchem solide Grundsätze verankert und Perspektiven erschlossen werden sollen, um die Entwicklung einer ausgewogeneren, solidarischeren Gesellschaft zu fördern, welche besser mit den ethischen Grundsätzen übereinstimmt, auf welche sich alle Akteure berufen: eine Gesellschaft der Kenntnisse und des geteilten Wissens, die auf gegenseitigem Zuhören und Solidarität gründet und dank dem fruchtbaren Dialog der Kulturen und Zivilisationen im Dienst einer Welt des Friedens steht.

Um die Diskussion zu vertiefen und die Resolutionen im Handlungsbereich zu verankern, sind Zeit und Geduld vonnöten. Wenn die Zivilgesellschaft sich auf die Errungenschaften der ersten Gipfelphase abstützt, hat sie gute Chancen, ihre Positionen, die weitgehend als fundiert anerkannt wurden, auf dem Konsensweg und im Geist der Partnerschaft durchzusetzen.

Wir bauen, trotz der unweigerlich auftretenden Probleme, gemeinsam an der Zukunft - geeint im schönen menschlichen Abenteuer. 